\title{
Mass loss, meridional circulation and turbulence in contemporary stellar evolution models
}

\author{
Suzanne Talon \\ Département de Physique, Université de Montréal, Montréal, PQ, H3C 3J7, Canada \\ email: talon@astro.umontreal.ca
}

\begin{abstract}
In this review, I will briefly discuss the hypotheses made in the treatment of modern rotating stellar models and review the expected efficiency of mixing along the HR diagram. The role of mixing in the localization of abundance anomalies will also be discussed. Finally, I will show how mass loss and gravitational settling of helium may influence the evolution of rotating stars, and how A stars can play a unique role in constraining our models.
\end{abstract}

Keywords. Turbulence, hydrodynamics, stars: abundances, stars: evolution, stars: chemically peculiar, Hertzsprung-Russell diagram, stars: interiors, stars: mass loss, stars: rotation

\section{Introduction}

Rotation plays an important role in determining stellar structure. It modifies surface properties such as the temperature and the local gravity and might even influence mass loss. Furthermore, it can lead to a significant amount of mixing due to meridional circulation and various hydrodynamical instabilities. The initial angular momentum contained in a star will thus be a third parameter guiding a star's structure and evolution. It is now widely recognized that a complete description of the evolution of the angular momentum distribution within the star is required and that rotational mixing is linked to the internal rotation profile.

The first modelization of this type was undertaken by Endal \& Sofia (1976, 1978, 1981) and pursued by Pinsonneault et al. (1989). However, in these early investigations meridional circulation was treated merely as a diffusive process.

The competition of meridional circulation and microscopic diffusion as competing processes in the formation of Am/Fm stars was quantified for the first time by Michaud (1982) and Charbonneau \& Michaud (1991). But the postulated solid body rotation was not derived from a self consistent calculation.

Here I will describe how meridional circulation can be described as a truly advective process $(\S 2)$ and present a modelization of anisotropic turbulent transport in stars $(\S 3)$. I will further describe the expected efficiency of mixing along the HR diagram $(\S 4)$, discuss the impact of various boundary conditions on this efficiency $(\S 5)$ and comment on the effect of mass loss on other physical processes $(\S 6)$. I will conclude with the examination of the constraints obtained from rotational mixing models of $\mathrm{A}$ and Am stars $(\S 7)$.

\section{Meridional circulation in 1D}

The evolution of the rotation state within a star subject to meridional circulation should in principle be treated at least with a $2 \mathrm{D}$ model. However, one can take advantage 
of the fact that in stratified fluids turbulence is expected to be strongly anisotropic. Assuming this is the case and that the horizontal turbulent viscosity $\nu_{h}$ is much larger than the vertical turbulent viscosity $\nu_{v}$ (or more specifically $\nu_{h} / l_{h}^{2} \gg \nu_{v} / l_{v}^{2}$ ) allows one to by-pass the difficulty. This approach was followed by Zahn (1992) to derive equations guiding the evolution of rotating stars. He assumes that the horizontal turbulence is efficient enough to enforce a shellular rotation state where $\Omega=\Omega(r)$.

A first order expansion leads to an advection-diffusion equation for the transport of angular momentum

$$
\rho \frac{\mathrm{d}}{\mathrm{d} t}\left[r^{2} \Omega\right]=\frac{1}{5 r^{2}} \frac{\partial}{\partial r}\left[\rho r^{4} \Omega U\right]+\frac{1}{r^{2}} \frac{\partial}{\partial r}\left[\rho \nu_{v} r^{4} \frac{\partial \Omega}{\partial r}\right]
$$

$\rho$ being the density and $\nu_{v}$ the vertical component of the turbulent viscosity. The amplitude of the vertical circulation velocity $u(r, \theta)=U(r)\left(P_{2}(\cos \theta)+1 / 5\right)$ is given by

$$
U(r)=\frac{L}{m g}\left(\frac{P}{C_{P} \rho T}\right) \frac{1}{\left(\nabla_{\mathrm{ad}}-\nabla+\nabla_{\mu}\right)}\left[E_{\Omega}+E_{\mu}\right],
$$

where $L$ is the luminosity, $m$ the mass, $P$ the pressure, $C_{P}$ the specific heat at constant pressure, $T$ the temperature, $\nabla_{\mathrm{ad}}=(\partial \ln T / \partial \ln P)_{\mathrm{ad}}, \nabla=\mathrm{d} \ln T / \mathrm{d} \ln P$, and $\nabla_{\mu}=$ $\mathrm{d} \ln \mu / \mathrm{d} \ln P$ : the adiabatic, radiative and mean molecular weight gradients, respectively (Maeder \& Zahn 1998). $E_{\Omega}$ and $E_{\mu}$, derived from first principles, depend on the rotation profile (neglecting any horizontal fluctuations) and on the horizontal variations of the mean molecular weight $\Lambda$, respectively (see Zahn 1992 and Maeder \& Zahn 1998 for details). Let us only remark that to first order $E_{\Omega} \propto \frac{\Omega^{2} R^{3}}{G M}$, which is a measure of the star's oblateness.

The presence of a strong horizontal turbulence $D_{h}$ modifies the evolution of chemicals. The combination of an advective meridional circulation with this horizontal turbulence is equivalent to considering an effective vertical diffusion given by

$$
D_{\text {eff }}=\frac{|r U(r)|^{2}}{30 D_{h}}
$$

(Chaboyer \& Zahn 1992). This equation expresses the fact that horizontal turbulence erodes the advective process and thus reduces the efficiency of meridional circulation in the transport of chemicals $\dagger$. The concentration of a given element $c_{i}$ then obeys

$$
\rho \frac{\mathrm{d} c_{i}}{\mathrm{~d} t}=\dot{c}_{i}+\frac{1}{r^{2}} \frac{\partial}{\partial r}\left[r^{2} \rho U_{i p} c_{i}\right] \frac{1}{r^{2}} \frac{\partial}{\partial r}+\left[r^{2} \rho\left(D_{\text {eff }}+D_{v}\right) \frac{\partial c_{i}}{\partial r}\right],
$$

where $\dot{c}_{i}$ is the nuclear production/destruction rate and $U_{i p}$ is the microscopic diffusion velocity of the element of interest with respect to protons. $D_{v}$ is the vertical turbulent diffusivity.

In this model, the rotation profile can reach a stationary state, in which the inward $\ddagger$ advection of angular momentum by meridional circulation balances the outward turbulent or viscous diffusion of momentum. This stationary state obeys

$$
U(r)=-\frac{5 \nu_{v}}{\Omega} \frac{\partial \Omega}{\partial r}
$$

$\dagger$ The advection of momentum is not affected since the horizontal average is performed on $r^{2} \Omega$ while turbulence homogenizes $\Omega$. See Chaboyer \& Zahn (1992) for details.

$\ddagger$ It can be shown that the advected flux will be directed inward as long as differential rotation is not too large. If differential rotation is large with the core rotating faster, the direction of the circulation reverses and both turbulent viscosity and meridional circulation carry angular momentum towards the surface. 
Calculations show that this equilibrium leads to a core rotating $\sim 1.2$ to 1.4 times faster than the surface in a homogeneous ZAMS star (this ratio rises in an evolved star). In a static model, there is thus no net momentum flux. However, there always exists a flux of concentration, as both the effective and turbulent diffusivity lead to concentration fluxes that are directed opposite to the concentration gradient. Moreover, while horizontal diffusion acts to reduce the efficiency of diffusion for the chemicals, it does not have the same effect on angular momentum; in a turbulent region, $D_{v} \simeq \nu_{v}$ is thus much larger than $D_{\text {eff }}$.

As the model star evolves, the circulation must adjust itself to provide the momentum flux required to reconstruct the equilibrium profile. This readjustment is made more drastic by mass loss, as the upward migration of the surface layers will create an even larger deficit of momentum. Mass loss is thus a key ingredient in the determination of the amount of large scale mixing taking place in the outer layers of stars. This is especially true of slow rotators in which turbulence is not large at the surface.

\section{Turbulence}

To complete the model, one has to obtain an estimate of the turbulent viscosities $\nu_{v}$ and $\nu_{h}$ and turbulent diffusivities $D_{v}$ and $D_{h}$. We will assume that $D_{v}=\nu_{v}$ and $D_{h}=\nu_{h}$. The source of turbulence considered here is the shear instability $\dagger$.

Shear instabilities develop because energy is stored in differential rotation. A linear stability analysis shows that instability may grow as soon as there is an inflection point in the profile (Rayleigh 1880, Watson 1981). However, laboratory experiments indicate that destabilization occurs even if this is not the case, and in general one may assume the presence of turbulence as soon as the Reynolds number is large enough (see, e.g., Richard \& Zahn 1999). This is the point of view we shall adopt.

In the case of the vertical viscosity $\nu_{v}$, work must be done against the stable stratification for the instability to occur. In the case relevant to stellar models, this will be made possible only if the thermal stratification measured by the Brunt-Väisälä frequency $N_{T}^{2}=\frac{g \delta}{H_{P}}\left(\nabla_{\text {ad }}-\nabla\right)$ is reduced by the thermal diffusivity (Townsend 1958, Dudis 1974, Lignières et al. 1999). Stratification in stars is also due to mean molecular weight gradients. The composition part of the Brunt-Väisälä frequency $N_{\mu}^{2}=\frac{g \phi}{H_{P}} \nabla_{\mu}=-g \frac{\mathrm{d} \ln \mu}{\mathrm{d} r}$ is unaffected by thermal diffusivity. However, it can be weakened through the action of strong horizontal diffusion (Talon \& Zahn 1997). Meynet \& Maeder (1997) have shown that such an effect has to be taken into account if rotational mixing is to account for abundance anomalies in massive stars.

Small eddies are easier to render unstable by these diffusive processes, but they lead to a limited amount of mixing. Very large eddies remain stable. A turbulent diffusion coefficient can be deduced which corresponds to the largest unstable eddies. Taking into account both thermal diffusivity and horizontal diffusion yields

$$
\nu_{v}=\frac{8 R i_{c}}{5} \frac{(r \mathrm{~d} \Omega / \mathrm{d} r)^{2}}{N_{T}^{2} /\left(K+D_{h}\right)+N_{\mu}^{2} / D_{h}} .
$$

for the vertical viscosity, where $R i_{c} \simeq 1 / 4$ is the critical Richardson number. The leading factor $8 R i_{c} / 5$ depends on the geometry (here assumed spherical) of the turbulent eddy and on the exact value of $R i_{c}$. It is thus not well constrained only from theory and could

$\dagger$ For a discussion on the magnitude of the baroclinic instability which will be neglected here, see, e.g., Spruit \& Knobloch (1984). 

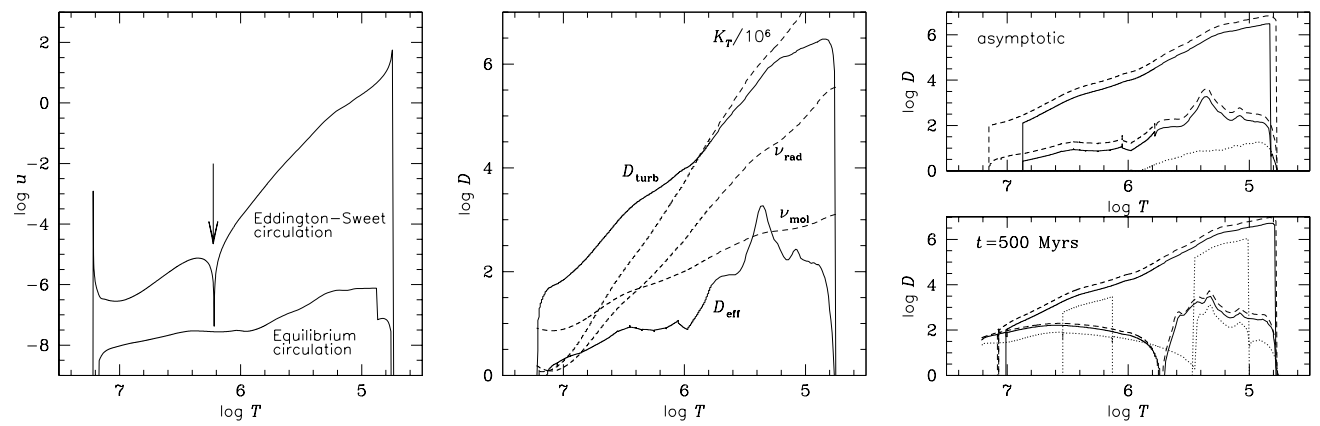

Figure 1. (left) Comparison of the Eddington-Sweet circulation velocity, including the reversed cell due to the Gratton-Öpik term (indicated by the arrow) and of the asymptotic solution described by (2.5), for a turbulent diffusion given by (3.1) and a surface velocity of $100 \mathrm{~km} \mathrm{~s}^{-1}$ in a $1.7 M_{\odot}$ star. The magnitude of the circulation is reduced by several orders of magnitudes, while the reversed cell disappears totally, since the circulation must transport momentum outward to balance the turbulent diffusion. (center) Turbulent and effective diffusion corresponding to the asymptotic rotation state for a surface velocity of $100 \mathrm{~km} \mathrm{~s}^{-1}$. (right) Turbulent and effective diffusion for various surface velocities (dashed - 150, full - 100, dotted - $50 \mathrm{~km} \mathrm{~s}^{-1}$ ) in the asymptotic state, and in an evolving model. These models include a cutoff based on the critical Reynolds number (cf. Eq. 3.2).

vary by a factor of a few either way. Furthermore, this instability will grow only provided

$$
\nu_{v} \gtrsim\left(\nu_{\mathrm{mol}}+\nu_{\mathrm{rad}}\right) R e_{c}
$$

where the critical Reynolds number is $R e_{c} \simeq 10$.

For horizontal shears there is no competing force preventing the growth of the instability. The magnitude of the horizontal turbulent viscosity is related to the magnitude of the horizontal differential rotation $\widehat{\Omega}$, which is constantly regenerated by meridional circulation. Zahn (1992) originally suggested that it should be proportional to the horizonal shear

$$
\nu_{h} \simeq \frac{1}{c_{h}} r|2 V-\alpha U|
$$

and that $c_{h}$ could be determined from observations, but it did not rely on a specific model. This prescription has recently been reviewed by Maeder (2003) and Mathis et al. (2004). The first paper proposes a formulation in which the viscosity is based on the dissipation and feeding of turbulent energy while the second relies on experimental results. Both lead to the same order of magnitude for $\nu_{h}$. However, the second formulation is smoother and will thus be preferred here:

$$
\nu_{h}=\left(\frac{\beta}{10}\right)^{1 / 2}\left(r^{2} \Omega\right)^{1 / 2}(r|2 V-\alpha U|)^{1 / 2}
$$

with $\beta \simeq 1.5 \times 10^{-5}$ derived from laboratory measures ( $\alpha$ is a measure of differential rotation). Here again, one may wonder whether this coefficient is correct for compressible fluids and a modification by a factor of a few cannot be ruled out a priori. Recent models by Maeder (private com.) suggest that this prescription is actually too strong and produces too little mixing in massive stars; a value between (3.3) and (3.4) should be preferred. This also suggest that horizontal differential rotation might have to be considered in future work (Mathis \& Zahn 2004). 


\section{Rotational mixing along the HR diagram}

To understand the varying importance of mixing along the HR diagram, one may discuss various time-scales involved with mixing. First, let us note that the time spent on the main sequence $\tau_{\mathrm{ms}}$ varies as $\tau_{\mathrm{ms}} \propto M^{-0.6}$ for $M \gtrsim 10 M_{\odot}$ and $\tau_{\mathrm{ms}} \propto M^{-3}$ for $M \lesssim 10 M_{\odot}$ (Schaller et al. 1992). The time-scale for turbulent mixing is of order

$$
\tau_{\text {turb }} \simeq \frac{R^{2}}{D}
$$

If turbulence is dominated by shears, as suggested here, then we have $D \propto K$. Classical scalings (see, e.g., Hansen \& Kawaler 1994) yield $R \propto M^{0.75}$ and $K \propto M^{3.25}$ for electron scattering opacities and $K \propto M^{5.3}$ for Kramers opacities. Thus, we get

$$
\tau_{\text {turb }} \propto M^{-1.75} \text { to } M^{-3.8}
$$

(Maeder 1998). This implies that the level of mixing (measured by $\tau_{\mathrm{ms}} / \tau_{\text {turb }}$ ) rises with mass above $\sim 10 M_{\odot}$ and stays about constant in lower mass stars for a given rotation profile. In the framework of rotational mixing, this explains why evidences of deep mixing are observed only in massive stars while "surface" abundance anomalies driven by microscopic diffusion occur in lower masses.

One must also consider the Eddington-Sweet time-scale, which gives an estimate of the time required to reach the asymptotic rotation profile defined by $(2.5)$

$$
\tau_{\text {E.S. }} \simeq \frac{R}{U} \propto \frac{1}{\alpha} \frac{M^{2}}{L R} .
$$

Using the relation $L \propto M^{2.25}$ for $M \gtrsim 10 M_{\odot}$ and $L \propto M^{4}$ for $M \lesssim 10 M_{\odot}$, we get

$$
\tau_{\text {E.S. }} \propto M^{-2.5} \text { for low mass } \propto M^{-0.75} \text { for high mass stars }
$$

for a given rotation velocity $V=\Omega R$. These time-scales vary with mass similarly to the main sequence lifetime.

For a $M=1.7 M_{\odot}$ star rotating at $100 \mathrm{~km} \mathrm{~s}^{-1}, \tau_{\mathrm{E} . \mathrm{S} .} \simeq 10 \% \tau_{\mathrm{ms}}$. Thus, for fast rotators, the initial rotation profile has little impact on the main sequence evolution. However, if the rotation velocity drops to $30 \mathrm{~km} \mathrm{~s}^{-1}$, we get $\tau_{\mathrm{E} . \mathrm{S}} \simeq \tau_{\mathrm{ms}}$. This has received little attention from theoreticians of rotational mixing so far; it implies that, in very slow rotators, turbulent diffusion should not drop as fast as $\Omega^{2}$.

Figure 2 illustrates the impact of the initial rotation state on the diffusion coefficient. Two cases are shown:

(a) asymptotic rotation state according to (2.5) on the main sequence;

(b) solid body rotation in a fully convective star at the top of the Hayashi track.

If meridional circulation and turbulence are the only processes contributing to the transport of momentum, condition (b) should apply. The initial rotation profile could be made somewhat smoother by including angular momentum transport by internal gravity waves, since the convective envelope is deep during the pre-main sequence phase (see Talon \& Charbonnel 2003) or some large scale magnetic field (see, e.g., MacGregor \& Brenner 1991). It would also be smoother in a star that completed its accretion phase after the formation of its radiative core (see, e.g., Stahler 1983). Condition (a) which would require strong coupling between the radiative and the convective zone during the pre-main sequence phase is not expected to be realistic from a theoretical point of view. Furthermore, recent observations of the evolution of the surface velocity from the birth line to the ZAMS favor core-envelope decoupling during the pre-main sequence as a general rule (Wolff, Strom \& Hillenbrand 2004). 

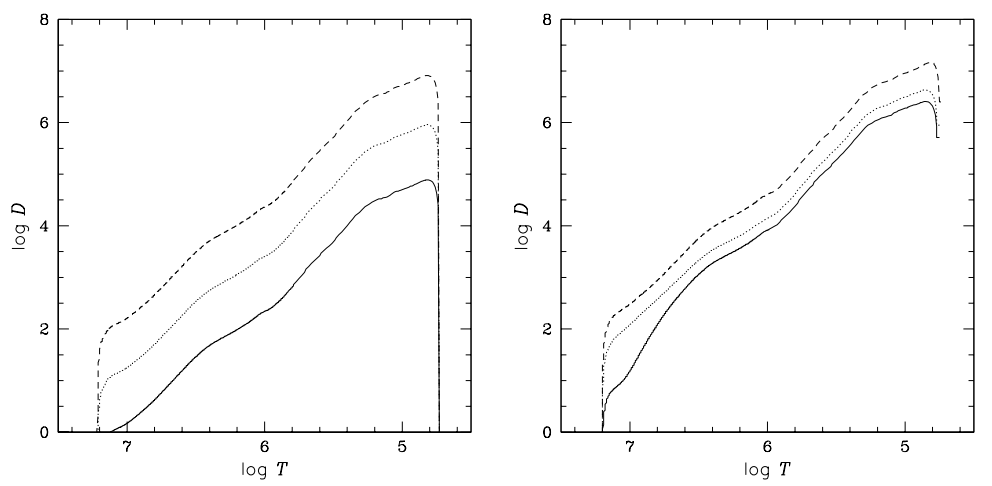

Figure 2. (left) The equilibrium turbulent diffusion profile on the ZAMS. (right) The turbulent diffusion profile at 500 Myrs with angular momentum evolution from the PMS. No cutoff based on the Reynolds number has been applied here ( $c f$. Eq. 3.2) and the velocities shown here are denoted by different lines (dashed - 150, dotted - 50, and full - $15 \mathrm{~km} \mathrm{~s}^{-1}$ ).

When differential rotation on the PMS is considered, mixing in slow rotators increases. This is related to the larger differential rotation, linked to the longer Eddington-Sweet time-scale. This could explain why abundance anomalies do not depend on the rotation velocity for slow rotators (see, e.g. Varenne \& Monier 1999).

\section{Boundary conditions}

To complete the mathematical system of equations, one must also specify boundary conditions. Since the problem is described by a fourth order differential equation, four conditions are required. The first two impose momentum conservation at convective boundaries

$$
\begin{array}{ll}
\frac{\partial}{\partial t}\left[\Omega \int_{r_{t}}^{R} r^{4} \rho \mathrm{d} r\right]=-\frac{1}{5} r^{4} \rho \Omega U+\mathcal{F}_{\Omega} & \text { for } r=r_{t} \\
\frac{\partial}{\partial t}\left[\Omega \int_{0}^{r_{b}} r^{4} \rho \mathrm{d} r\right]=\frac{1}{5} r^{4} \rho \Omega U & \text { for } r=r_{b}
\end{array}
$$

where $r_{t}$ and $r_{b}$ are respectively the top and the bottom of the convection zone, which are assumed to rotate as solid bodiest. Here, we also allow explicitly for a torque $\mathcal{F}_{\Omega}$ to be applied at the stellar surface, as would be the case in an asynchronous binary system, or in a star subject to braking due to a magnetic wind. If such braking occurs, it will lead to an increase of both meridionnal circulation and shear turbulence and enhance significantly mixing close to the surface. For A stars, we will assume $\mathcal{F}_{\Omega}=0$.

For the other two conditions,

$$
\frac{\mathrm{d} \Omega}{\mathrm{d} r}=0 \quad \text { for } r=r_{t}, r_{b}
$$

has generally been used. This condition actually states that the differential rotation just below the convection zone should be the same as in the convection zone; in a 1D model, it is equivalent to the stress-free condition of hydrodynamics. However, it is unclear whether there is no radial differential rotation at the boundary of convective regions, and thus the impact of the choice of boundary condition should also be examined.

$\dagger$ This is motivated by the fact that the Sun presents little differential rotation in radius in its convection zone. 


\section{Mass loss}

Another parameter to take into account in modeling is the presence of some amount of mass loss at the stellar surface. If the star looses some mass, the inner layers will constantly have to move outward to replace those that were lost to the stellar wind. This will have two impacts on the transport of chemicals:

- Firstly, the homogeneous drift of those surface layers will reduce the efficiency of element separation by microscopic diffusion;

- Secondly, rotational mixing will be enhanced by the required transport of momentum to the surface layers that are spun down by local angular momentum conservation as they drift upward.

As far as theory is concerned, the nature of mass loss in A stars is not well understood. It should not come from a hot corona as in the case of the Sun (Parker 1960) due to the absence of a deep surface convection zonet; radiative accelerations are also believed to be too small to produce significant mass loss (Abbott 1982) in main sequence A stars. Babel (1995) studied the case of A stars in more details. He concludes that, for an effective temperature between $8000 \lesssim T_{\text {eff }} \lesssim 14000 \mathrm{~K}$, only metals can be lifted by radiative forces, giving rise to fully seperated winds, consisting only of metals. From complete calculations made for an A star at $10000 \mathrm{~K}$, he finds a wind of $\dot{M} \lesssim 10^{-16} M_{\odot} \mathrm{yr}^{-1}$. Such winds would lead to very different anomalies, as the star could be drained only of some metals (Michaud \& Charland 1986).

From an observational viewpoint, mass loss is very difficult to measure directly in A stars. Bertin et al. (1995) derived a mass loss rate in Sirius A between $2 \times 10^{-13}$ and $1.5 \times 10^{-12} M_{\odot} \mathrm{yr}^{-1}$ based on $\mathrm{Mg}$ II lines. However, only the lower limit would permit surface abundance anomalies to form; for higher mass loss rates, matter would leave the surface of the star before microscopic diffusion had time to act (Babel \& Michaud 1991). It has been suggested that surface abundance anomalies in A stars could represent a means to measure mass loss rates, if they are produced solely by the competition of mass loss and microscopic diffusion (see, e.g., Landstreet et al. 1998). Babel (1993) suggests that, in Ap stars, inhomogeneous mass loss modulated by surface magnetic fields can be responsible for "patches" in the surface abundance of various elements.

\section{Observational constraints from Am stars}

Rotation plays an important role in the appearance of the Am phenomenon. Indeed, only slow rotators bear the characteristic signature of radiative forces and settling (see, e.g., the review by Conti (1970)). Furthermore, it seems that slow rotation (below $100 \mathrm{~km} \mathrm{~s}^{-1}$ ) is a necessary and sufficient condition for late Am stars, and a necessary (but not sufficient) condition for early Am stars.

This must be reflected in the rotating models and give strong constraints on the coefficients for $D_{v}$ (cf. Eq. 3.1) and $D_{h}$ (cf. Eq. 3.4) as well as on the initial rotation profile (cf. Fig. 2).

\subsection{Meridional circulation and mixing in A stars}

The efficiency of rotational mixing changes during the main sequence. It is initially rather large, since the pre-main sequence contraction leads to the formation of a rapidly rotating core. This phase lasts for an Eddington-Sweet time-scale (4.3). After the asymptotic state is reached, differential rotation in the stellar interior rises between the ZAMS and the TAMS. This is related to the increase of the ratio of stellar luminosity to surface gravity

$\dagger$ Recent observational evidence indicate there could be some activity in late A stars. 
during the same period (see Eq. 2.2) which induces an enhancement of the meridional circulation. We thus expect the presence of a minimum in the efficiency of rotational mixing occurring at $t=\tau_{\text {E.S. }}$.

Another delicate issue to consider is the role of mean molecular weight gradients. It is well known that these gradients act to reduce the growth of instabilities, leading to a reduction of the turbulent diffusion coefficients presented here. This could be an important ingredient in the formation of Am stars.

Another point that has been neglected here is the role of horizontal diffusion in the reduction of the efficiency of vertical turbulence in the transport of chemicals ( $c f$. Vincent et al. 1996). The importance of this effect will be discussed in a future study (Talon, Richard \& Michaud, in preparation).

\section{Acknowledgements}

S.T. is supported by NSERC of Canada.

\section{References}

Abbott, D. C., 1982 ApJ 259, 282.

Babel, J., 1983 In Peculiar versus Normal Phenomena in A-type and Related Stars, IAU Coll. 138, PASP, ed. M.M. Dworetsky, F. Castelli, R. Faraggiana, 458.

Babel, J., 1995 A $\& A$ 301, 823.

Babel, J. \& Michaud, G., 1991 ApJ 241, 493.

Bertin, P., Lamers, H.J.G.L.M., Vidal-Madjar, A., Ferlet, R. \& Lallement, R., 1995 A 8 A 302, 899.

Chaboyer, B. \& Zahn, J.-P., 1992 A $\& A$ 253, 173.

Charbonneau, P. \& Michaud, G., 1991 ApJ 370, 693.

Conti, P. S., 1970 PASP 82, 488.

Dudis, J. J., 1974 J. Fluid Mech. 64, 65.

Endal, A. S. \& Sofia, S., 1976 ApJ 210, 184.

Endal, A. S. \& Sofia, S., 1978 ApJ 220, 279.

Endal, A. S. \& Sofia, S., 1981 ApJ 243, 625.

Hansen, C. J. \& Kawaler S. D. , 1994 Stellar Interiors Ed. Springer-Verlag, A\&A Library.

Landstreet, J. D., Dolez, N. \& Vauclair, S., 1998 A $\& A$ 333, 977.

MacGregor, K. B. \& Brenner, M., 1991 ApJ 376, 204.

Maeder, A., 1998 In Properties of Hot Luminous Stars, ASP Conf. Series, 131, ed. Ian D. Howarth, 85.

Maeder, A., 2003 A\&A 399, 263.

Maeder, A. \& Zahn, J.-P., 1998 A $\& A$ 333, 977.

Mathis, S., Palacios, A. \& Zahn, J.-P., $2004 A \& A$ submitted.

Meynet, G. \& Maeder, A., 1997 A $\& A$ 321, 465.

Michaud, G., 1982 ApJ 258, 349.

Michaud, G. \& Charland, Y., 1986 ApJ 311, 326.

Pinsonneault, M. H., Kawaler, S. D., Sofia, S. \& Demarque, P., 1989 ApJ 338, 424.

Rayleigh, Lord, 1880 Proc. London Math. Soc. 11, 57.

Richard, D. \& Zahn, J.-P., 1999 A $\& A$ 347, 734.

Schaller, G., Schaerer, D., Meynet, G. \& Maeder, A., 1992, A\&̈AS 96, 269.

Stahler, S. W., 1983 ApJ 822, 274.

Spruit, H. C. \& Knobloch, E., 1984 A\&A 132, 89.

Talon, S. \& Charbonnel, C., 2003 A $\& A$ 405, 1025.

Talon, S. \& Zahn, J.-P., 1997 A $\& A$ 317, 749.

Towsend, A. A., 1958 J. Fluid Mech. 4, 361.

Varenne, O. \& Monier, R., 1999 A $\& A$ 351, 247.

Vincent, A., Michaud, G. \& Meneguzzi, M., 1996 Phys. Fluids 8 (5) 1312. 
Watson, M., 1981 Geophys. Astrophys. Fluid Dyn. 161, 285.

Wolff, S. C., Strom, S. E. \& Hillenbrand, L. A., 2004 ApJ 601, 979.

Zahn, J.-P., 1992 AEA 265, 115.

\section{Discussion}

KHALACK: Have you investigated the influence of the magnetic field on the shellular structure of a modelled star? How will such a star evolve?

TALON: The set of equations presented here does not take into account internal magnetic forces. However, one can take into account the combined effect of mass loss and the surface magnetic field that will act to brake the star's surface. This leads to an increase of the internal (surface) differential rotation, and thus, produces extra mixing.

KuBÁt: How could the stellar oblateness of rapidly rotating stars affect the processes in stellar envelopes?

TALON: The oblateness becomes significant (of order 10\%) for stars rotating faster than about $150 \mathrm{~km} \mathrm{~s}^{-1}$. In that case, the temperature of the atmosphere will vary accoring to latitude. 